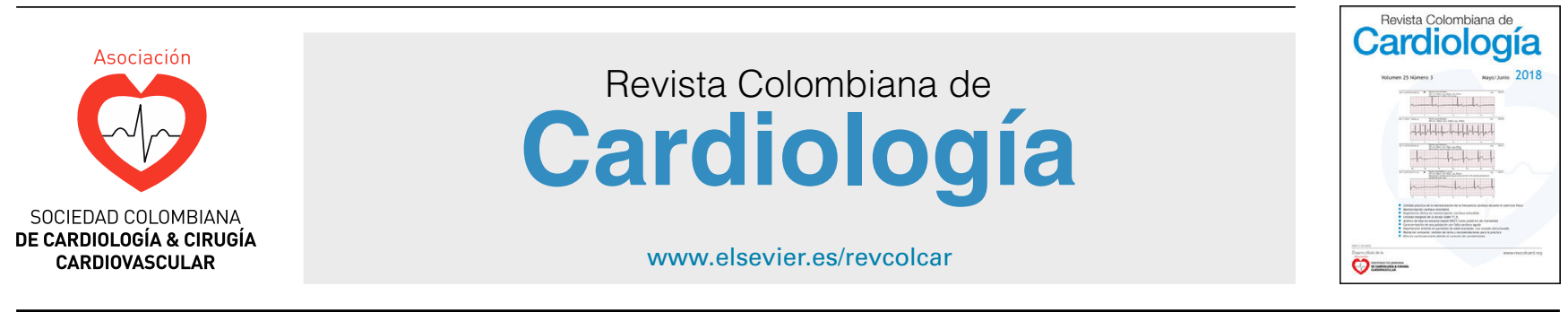

CARDIOLOGÍA DEL ADULTO - ARTÍCULO ORIGINAL

\title{
Inhibidores de la enzima convertidora de angiotensina y bloqueadores de los receptores de angiotensina II: ¿aumentan el riesgo de padecer COVID-19?
}

\author{
Dora Inés Molina ${ }^{\mathrm{a}, \mathrm{b}, *}$, Tania Marcela Muñoz ${ }^{\mathrm{a}}$ y Katterine Guevara ${ }^{\mathrm{a}}$ \\ a Facultad de Ciencias para la Salud. Departamento Clínico, Universidad de Caldas, Manizales, Colombia \\ ${ }^{\mathrm{b}}$ Asociación IPS médicos internistas de Caldas, Manizales, Colombia
}

Recibido el 13 de mayo de 2020; aceptado el 25 de mayo de 2020

Disponible en Internet el 12 de junio de 2020

\author{
PALABRAS CLAVE \\ COVID-19; \\ SARS-CoV-2; \\ ACE2; \\ BRA; \\ IECA
}

\begin{abstract}
Resumen Un nuevo coronavirus, llamado coronavirus 2 del síndrome respiratorio agudo severo (SARS-CoV-2), se descubrió en diciembre de 2019 en Wuhan, China; el virus se intensificó rápidamente y el 11 de marzo de 2020 la Organización Mundial de la Salud lo declaró pandemia.

Los datos emergentes sugieren que los pacientes mayores con COVID-19 asociado a otras afecciones comórbidas, como diabetes, hipertensión, y enfermedades cardíacas y pulmonares, son, en particular, más susceptibles, en comparación con las poblaciones generales y tienen mayor mortalidad. Aún no está claro si esta mayor asociación de hipertensión arterial con COVID-19 y el mayor riesgo de mortalidad están directamente relacionados con la hipertensión arterial u otras comorbilidades asociadas, o con el tratamiento antihipertensivo.

$\mathrm{Si}$ bien el mecanismo patogénico subyacente que une la hipertensión y la gravedad de la infección por COVID-19 aún no se ha dilucidado, se ha planteado la hipótesis de que la activación excesiva del sistema renina-angiotensina (RAS) podría contribuir a la progresión de la lesión pulmonar relacionada con COVID-19.

La preocupación sobre si los bloqueadores del receptor de angiotensina II (BRA) y los inhibidores de la enzima convertidora de angiotensina (IECA) pueden tener efectos nocivos sobre la morbilidad y la mortalidad de los pacientes con COVID-19 se basa en la especulación de que estos medicamentos aumentarían la regulación de la enzima convertidora de angiotensina II (ACE2), un receptor para el SARS-CoV-2, que aumentaría la carga viral y la lesión pulmonar.

Los estudios recientes concuerdan con las recomendaciones de las sociedades científicas que plantean evitar la suspensión o cambio de medicación antihipertensiva, pues no hay evidencia que muestre que estos puedan ser tomados como factores de riesgo para gravedad o mortalidad por COVID-19.

(C) 2020 Sociedad Colombiana de Cardiología y Cirugía Cardiovascular. Publicado por Elsevier España, S.L.U. Este es un artículo Open Access bajo la licencia CC BY-NC-ND (http:// creativecommons.org/licenses/by-nc-nd/4.0/).
\end{abstract}

\footnotetext{
* Autor para correspondencia.

Correo electrónico: doraines56@gmail.com (D.I. Molina).
} 


\section{KEYWORDS \\ COVID-19; \\ SARS-CoV-2; \\ ACE2; \\ BRA; \\ ACEI}

\section{Angiotensin converting enzyme inhibitors and angiotensin receptor blocker: do they} increase the risk of COVID-19?

Abstract A new coronavirus, called severe acute respiratory syndrome coronavirus 2 (SARSCoV-2), was discovered in December 2019 in Wuhan, China; the virus escalated rapidly and on March 11, 2020, the World Health Organization declared it a pandemic.

Emerging data suggests that older patients with COVID-19 associated with other comorbid conditions such as diabetes, hypertension, heart and lung diseases are particularly more susceptible, compared to general populations, and have higher mortality. It is not yet clear whether this increased association of high blood pressure with COVID-19 and the increased risk of mortality are directly related to high blood pressure or other associated comorbidities, or to antihypertensive treatment.

Although the underlying pathogenic mechanism linking hypertension and severity of COVID-19 infection remains to be elucidated, it has been hypothesized that excessive activation of the renin-angiotensin system (RAS) could contribute to the progression of COVID-19 related lung injury.

Concern about whether angiotensin II receptor blockers (ARBs) and angiotensin converting enzyme (ACE) inhibitors may have deleterious effects on morbidity and mortality in patients with COVID-19 is based on speculation that these drugs would increase the regulation of angiotensin II converting enzyme (ACE2), a receptor for SARS-CoV-2, which would increase viral load and lung damage.

Recent studies are consistent with the recommendations of scientific societies that propose avoiding the suspension or change of antihypertensive medication, as there is no evidence that shows that these can be taken as risk factors for severity or mortality from COVID-19.

(c) 2020 Sociedad Colombiana de Cardiología y Cirugía Cardiovascular. Published by Elsevier España, S.L.U. This is an open access article under the CC BY-NC-ND license (http:// creativecommons.org/licenses/by-nc-nd/4.0/).

\section{Introducción}

Un nuevo coronavirus, llamado coronavirus 2 del síndrome respiratorio agudo severo (SARS-CoV-2), se descubrió en diciembre de 2019 en Wuhan, China. Se intensificó rápidamente y el 11 de marzo de 2020 la Organización Mundial de la Salud lo declaró una pandemia. El espectro clínico de COVID-19 varía desde infección asintomática de las vías respiratorias superiores, hasta neumonía grave asociada con el síndrome de dificultad respiratoria aguda (SDRA) ${ }^{1}$.

En la actualidad, la investigación se enfoca especialmente en comprender por qué las personas son hospitalizadas, reciben cuidados intensivos y con frecuencia mueren como consecuencia de SARS-CoV- $2^{3}$. Los datos emergentes sugieren que los pacientes mayores con COVID-19 con otras afecciones comórbidas, como diabetes, hipertensión, enfermedades cardíacas y pulmonares, son, en particular, más susceptibles, en comparación con la población general y tienen mayor mortalidad. La asociación de hipertensión arterial y diabetes en pacientes con COVID-19 no es inesperada, dada la creciente prevalencia de estas dos enfermedades crónicas en el mundo ${ }^{4}$.

La comprensión reciente del papel de la desregulación inmune en la hipertensión puede proporcionar un posible vínculo entre la desregulación inmune y un curso más grave de COVID-19. El rápido deterioro en pacientes con COVID-19 se asocia con una tormenta de citoquinas proinflamatorias; en consecuencia, en pacientes con COVID-19 se ha observado un aumento en la interleucina 2 (IL) -2, IL-6 e
IL-7, factor estimulante de colonias de granulocitos, quimiocina CXC 10 (CXCL10) y factor de necrosis tumoral $\alpha$ $(\mathrm{TNF}-\alpha)$. Curiosamente, las mismas citocinas se han asociado con el desarrollo de la hipertensión en estudios experimentales y clínicos observacionales, así como en estudios intervencionistas ${ }^{5}$, sin embargo, el tema de hipertensión y COVID-19 se sale de los objetivos del artículo por lo que no se ahondará en este.

Aún no está claro si esta mayor asociación de hipertensión arterial con COVID-19 y el mayor riesgo de mortalidad están directamente relacionados con la hipertensión arterial u otras comorbilidades asociadas, o con el tratamiento antihipertensivo. Ha habido una creciente preocupación de que esta asociación pueda verse confundida por el tratamiento con ciertos medicamentos antihipertensivos como los bloqueadores del sistema renina-angiotensina (RAS).

El objetivo de este artículo es revisar de la evidencia científica disponible sobre la asociación entre el uso hospitalario de inhibidores de la enzima convertidora de angiotensina (IECA)/bloqueadores del receptor de angiotensina II (BRA) y el riesgo de resultados adversos en COVID-19 entre pacientes con hipertensión.

\section{Metodología}

Se realizó una búsqueda bibliográfica en abril de 2020 en PubMed utilizando los descriptores: COVID-19, SARS-COV-2, ACE2, BRA, ACEI. También se identificaron referencias de artículos de revisión relevantes, así como de la sección de artículos similares de PubMed. 


\section{Visión general}

Los primeros estudios se iniciaron principalmente en cohortes chinas, ya que China es el presunto punto de origen de COVID-19. Estos estudios indican que los pacientes sometidos a unidades de cuidados intensivos ( $\mathrm{UCI}$ ) con frecuencia muestran hipertensión, diabetes y enfermedades cardiovasculares, o ambas. Estos pacientes demuestran tasas de mortalidad mucho más altas en comparación con los pacientes que están libres de estas comorbilidades ${ }^{3}$.

Si bien el mecanismo patogénico subyacente que une la hipertensión y la gravedad de la infección por COVID-19 aún no se ha dilucidado, se ha planteado la hipótesis de que la activación excesiva del RAS podría contribuir a la progresión de la lesión pulmonar, al promover la respuesta inflamatoria y la tormenta de citoquinas, estimular el sistema de oxidasa NADH/NADPH y desencadenar vasoconstricción.

La renina, la angiotensina y la aldosterona representan el núcleo de un eje hormonal complejo, que contribuye al control de la presión arterial, la reabsorción de sodio, la inflamación y la fibrosis. El desequilibrio o modificación de RAS puede desencadenar o tratar muchas enfermedades, incluyendo insuficiencia cardíaca, hipertensión, diabetes y aterosclerosis ${ }^{7}$.

El angiotensinógeno es liberado principalmente por el hígado y luego es escindido por la renina, que es secretada por las células yuxtaglomerulares en el riñón, generando así el decapéptido angiotensina I (Ang I). Ang I se convierte en angiotensina II (Ang II) mediante enzimas convertidoras de angiotensina (ECA), expresadas por las células endoteliales de varios órganos, como pulmón, corazón, riñón y cerebro. Ang II es la molécula más relevante de la vía RAS y realiza su función activando los siguientes receptores acoplados a la proteína G: receptor de angiotensina II tipo 1 (AT1R) y receptor de angiotensina II tipo 2 (AT2R). Los efectos ejercidos por estos dos receptores de membrana son opuestos, en particular, AT1R induce efectos perjudiciales, como inflamación, fibrosis y equilibrio redox alterado, además de propiedades vasoconstrictoras, mientras que AT2R está involucrado en acciones protectoras y regeneradoras (antiinflamatorio, antifibrótico, metabólico) y en la liberación de moléculas vasodilatadoras. Por lo tanto, el punto de equilibrio del RAS está representado por Ang II, que también se puede convertir en heptapéptido Ang- (1-7) gracias a la acción de la enzima convertidora de angiotensina 2 (ECA2). Ang- (1-7), que también puede generarse por la escisión de Ang I por endopeptidasas, y une los receptores que contrarrestan la mayoría de las acciones nocivas del eje ECA / Ang II / AT1, especialmente en condiciones patológicas. Los IECA inhiben la formación de angiotensina II y, en consecuencia, los efectos desencadenados por su interacción con los receptores AT1R (vasoconstricción, retención de sodio y agua, activación simpática, crecimiento celular) y receptores $A T 2 R^{8}$.

\section{Relación entre la enzima convertidora de angiotensina II y la infección por SARS-CoV-2}

La entrada del coronavirus a la célula es facilitada por la proteína espiga ( $\mathrm{S}$ ). Las proteínas $\mathrm{S}$ de diferentes coronavirus pueden utilizar diferentes receptores; MERS utiliza CD26, mientras que el SARS CoV y el SARS CoV2 utilizan la enzima ECA-2. La eficiencia de la interacción entre la proteína $\mathrm{S}$ y la ECA-2 puede ser un determinante clave de la transmisibilidad del virus, la replicación viral y la gravedad de la enfermedad. En teoría, esta eficiencia podría verse influenciada por cambios o sustituciones de aminoácidos en la proteína $S$ viral o en el receptor ECA-2 en la célula huésped ${ }^{4}$.

La infección por SARS-CoV 2 regula a la baja la expresión superficial de la proteína de unión, ECA-2, un componente fundamental para la entrada a la célula huésped como se mencionó anteriormente. En particular, la baja expresión de ECA-2 se asocia con una mayor gravedad del fenotipo en los estudios in vitro de células epiteliales de las vías respiratorias humanas. Kuba et al. demostraron que la proteína espiga de SARS-CoV aumenta la expresión de Ang II y la regulación negativa de ECA-2, con la lesión pulmonar resultante ${ }^{7}$.

El bloqueo del AT1R regula al alza la ECA-2 a través de un mecanismo de retroalimentación negativa, y sirve como mecanismo de protección pulmonar a través de la mayor conversión de Ang II a Ang- (1-7), amortiguando efectivamente la lesión pulmonar del virus. Estos hallazgos han llevado a los investigadores a postular que la vía ECA-2 / Ang(1-7) puede servir como un objetivo terapéutico para combatir los efectos patológicos de Ang II. Haga et al. demostraron que la unión de SARS-CoV a ECA-2 induce el desprendimiento de ECA-2 como una forma soluble en el suero, y otros estudios validaron esos hallazgos. Además, la endocitosis de la proteína ECA-2 ocurre después de unirse al SARS-CoV, lo que disminuye aún más la actividad de ECA- $2^{7}$.

El hecho de que la hipertensión y otras formas de enfermedad cardiovascular que también se encuentran con frecuencia en pacientes con COVID-19, a menudo se tratan con IECA y BRA, ha planteado preguntas sobre la posibilidad de que estos agentes puedan ser beneficiosos o perjudiciales en pacientes tratados con ellos respecto a la susceptibilidad a adquirir COVID-19 o en relación con sus resultados clínicos 9 .

\section{Inhibidores de la enzima convertidora de angiotensina y bloqueadores de los receptores de angiotensina II en COVID-19}

La preocupación sobre si los BRA y los IECA pueden tener efectos nocivos sobre la morbilidad y la mortalidad de los pacientes con COVID-19 se basa en la especulación de que estos medicamentos aumentarían la regulación de ECA-2. Sin embargo, la evidencia de la regulación positiva de ECA-2 solo está limitada en estudios en animales que usan dosis relativamente altas de BRA e IECA. Además, no está claro si el aumento de ECA-2 inducido por BRA / IECA en realidad aumenta la susceptibilidad al SARS-CoV-2 o SAR-CoV. Es importante destacar que no existen estudios clínicos que respalden que los BRA y los IECA aumentan la susceptibilidad a la infección y empeoran los resultados cardiovasculares y de todas las causas en pacientes con COVID-19. Por lo tanto, la hipótesis subyacente a la preocupación no se extrapolaría fácilmente a los humanos, particularmente a los pacientes con COVID-191.

Como se mencionó anteriormente, la ECA-2 forma Ang (1-7) a partir de angiotensina II, y, por consiguiente, reduce 
la acción inflamatoria de la misma, y aumenta el potencial de los efectos antiinflamatorios de Ang (1-7). En consecuencia, al reducir la formación de Ang II en el caso de los IECA, o al antagonizar la acción de la angiotensina II al bloquear los AT1R en el caso de los BRA, estos agentes podrían contribuir a reducir la inflamación sistémica y particularmente en los pulmones, el corazón y los riñones. En consecuencia, los IECA y los BRA podrían disminuir el potencial de desarrollo SDRA, miocarditis o daño renal agudo, que puede ocurrir en pacientes con COVID-19; sin embargo, ninguna de estas posibilidades se ha demostrado en pacientes aún? .

Un estudio publicado en JAMA mostró que los IECA y BRA no tuvieron ningún efecto sobre la gravedad o el resultado de los pacientes con hipertensión hospitalizados por COVID-19. Juyi Li et al. analizaron datos de 1.178 pacientes con COVID19 hospitalizados en el Hospital Central de Wuhan del 15 de enero al 15 de marzo de 2020. De los pacientes, 30.7\% tenían hipertensión, y de estos, $31.8 \%$ estaban tomando IECA / BRA. El porcentaje de pacientes con hipertensión que tomaban IECA / BRA no difirió entre aquellos con infecciones severas y no graves (32.9 vs. $30.7 \% ; p=0.65)$ ni difirió entre no sobrevivientes y sobrevivientes $(27.3 \text { vs. } 33.0 \% \text {; } p=0.34)^{6}$.

En un estudio de casos y controles basado en la población en la región italiana de Lombardía, un total de 6.272 pacientes de casos en los que se confirmó la infección por SARS-CoV-2 entre el 21 de febrero y el 11 de marzo de 2020, fueron emparejados con 30.759 beneficiarios del Servicio Regional de Salud (controles). El uso de IECA y BRA fue más común entre los pacientes de casos que entre los controles, y los pacientes de casos tenían un peor perfil clínico. El uso de BRA o IECA no mostró ninguna asociación con COVID-19 entre los pacientes en general o entre pacientes que tuvieron un curso grave o mortal de la enfermedad ${ }^{10}$.

En un análisis observacional en una cohorte de más de 12.500 pacientes a quienes se les realizó la prueba de COVID19 en una gran red de salud en la ciudad de Nueva York, el tratamiento previo con medicamentos que actúan sobre el RAS no se asoció con un mayor riesgo de resultados positivos para COVID-19 ni hubo un riesgo sustancialmente mayor de COVID-19 grave asociado con cualquiera de los medicamentos estudiados entre los pacientes con una prueba positiva en dicha cohorte ${ }^{11}$.

En una preimpresión reciente, Liu et al. en un estudio retrospectivo de 511 pacientes con COVID-19 (edad mayor a 65 años) e hipertensión, los pacientes se clasificaron según el régimen antihipertensivo domiciliario. Los pacientes con BRA presentaron una disminución significativa de las probabilidades de desarrollar enfermedad grave por COVID-19 en el análisis univariado (OR 0.34, p 0.025) y en el análisis multivariable (OR 0.25, p 0.046), mientras que ninguno de los otros medicamentos demostró mejores resultados. Dos estudios retrospectivos posteriores han demostrado un efecto protector del bloqueo RAS, incluidas reducciones significativas en la carga viral ${ }^{7}$.

Kuba et al. encontraron que el losartán mostró una lesión pulmonar y un edema pulmonar significativamente disminuidos después de una lesión pulmonar aguda inducida por aspiración ácida (con la adición de la proteína de pico de SARS-CoV) en comparación con el placebo. De manera similar, tanto las infusiones de ECA-2 humano recombinante, como el losartán en ratones knock out con ECA-2 previnieron la lesión pulmonar grave y el edema pulmonar. Además, un análisis retrospectivo encontró tasas reducidas de muerte e intubación endotraqueal en pacientes con neumonía viral que continuaron con IECA ${ }^{4}$.

La evidencia sobre COVID-19 se acumula cada día. Se esperan estudios clínicos a gran escala con evidencia convincente, no solo estudios controlados aleatorizados, sino también grandes análisis de datos, así como estudios experimentales, para resolver la cuestión de si los BRA y los IECA tienen efectos neutrales, favorables o perjudiciales sobre la susceptibilidad al SRAS -CoV-2 y la gravedad y los resultados de COVID-191.

Por otro lado, es importante mencionar que en el contexto de la pandemia de COVID-19, algunos autores estuvieron tentados a especular que los bloqueadores de RAS aumentarían la infectividad del SARS-CoV-2 y la gravedad del curso clínico. Por tanto, sugirieron evitar los bloqueadores RAS durante la pandemia de COVID-195 ${ }^{5}$, pero los estudios muestran que, pacientes con insuficiencia cardíaca, infarto de miocardio previo, otras enfermedades cardiovasculares y enfermedad cerebrovascular, reciben beneficio del manejo con BRA e IECA en cuanto a mejoría de la calidad de vida y los resultados de supervivencia, mientras que la interrupción conduce a un nuevo inicio o exacerbación de la insuficiencia cardíaca, enfermedad cardiovascular y accidente cerebrovascular y el empeoramiento del pronóstico. Por lo tanto, los inhibidores de RAS deben continuarse en pacientes de alto riesgo que hayan recibido terapia médica dirigida por pautas. No es raro que el cambio de una a otra clase de agentes antihipertensivos genere la desestabilización de la presión arterial y requiera visitas frecuentes para la titulación del fármaco aumentando el riesgo de contagio por SARS-CoV-2 debido a la mayor exposición ${ }^{1}$.

En consecuencia, incluso en pacientes con hipertensión controlada de forma estable sin indicaciones convincentes, se cree que no solo suspender el tratamiento, sino también cambiar de BRA o IECA a otros agentes antihipertensivos se debe evitar, a menos que los médicos tomen una decisión basada en consideraciones específicas ${ }^{1}$, así como lo menciona la declaración publicada conjuntamente por la Asociación Americana del Corazón (AHA), la Sociedad Americana de Falla Cardiaca (HFSA), el Colegio Americano de Cardiología (ACC) y la Sociedad Internacional de Hipertensión (ISH), con sus siglas respectivas en inglés.

\section{Conclusión}

Las sociedades científicas y los expertos se han pronunciado unánimemente al aconsejar que los pacientes no deben suspender la terapia con IECA o BRA por temor a que tengan un mayor riesgo de infección, enfermedad grave o muerte durante la pandemia de COVID-19. Los estudios mencionados en esta revisión respaldan estas recomendaciones y dan pie para plantear investigaciones que evalúen el beneficio del bloqueo del eje Angll-AT1R.

Hay muchas otras ideas de investigación que permitirán realizar más estudios en los próximos meses con el objetivo de aclarar mejor cualquier estrategia terapéutica contra el SARS-CoV-2, y el RAS probablemente jugará un papel como protagonista en este importante desafío. 


\section{Confidencialidad de los datos}

Los autores declaran que en este artículo no aparecen datos de pacientes.

\section{Derecho a la privacidad y consentimiento informado}

Los autores declaran que en este artículo no aparecen datos de pacientes.

\section{Conflicto de intereses}

Los autores declaran no tener ningún conflicto de intereses.

\section{Bibliografía}

1. Kai H, Kai M. Interactions of coronaviruses with ACE2, angiotensin II, and RAS inhibitors-lessons from available evidence and insights into COVID-19. Hypertens Res [Internet]. 2020:1-7. Disponible en: http://www.nature.com/articles/s41440-0200455-8.

2. Rico-Mesa JS, White A, Anderson AS. Outcomes in Patients with COVID-19 Infection Taking ACEI/ARB. Curr Cardiol Rep. 2020;22:20-3

3. Groß S, Jahn C, Cushman S, Bär C, Thum T. SARS-CoV-2 receptor ACE2-dependent implications on the cardiovascular system: From basic science to clinical implications. J Mol Cell Cardiol [Internet]. 2020. Disponible en: http://www.ncbi.nlm.nih.gov/pubmed/32360703.

4. Singh AK, Gupta R, Misra A. Comorbidities in COVID-19: Outcomes in hypertensive cohort and controversies with renin angiotensin system blockers. Diabetes Metab Syndr Clin Res Rev [Internet]. 2020;14:283-7. Disponible en: https://doi.org/10.1016/j.dsx.2020.03.016.

5. Kreutz R, Algharably EAE-H, Azizi M, Dobrowolski P, Guzik T, Januszewicz A, et al. Hypertension, the renin-angiotensin system, and the risk of lower respiratory tract infections and lung injury: implications for COVID-19. Cardiovasc Res. 2020:1-12.

6. Zhang P, Zhu L, Cai J, Lei F, Qin J-J, Xie J, et al. Association of inpatient use of angiotensin converting enzyme inhibitors and angiotensin ii receptor blockers with mortality among patients with hypertension hospitalized with COVID-19. Circ Res. 2020, http://dx.doi.org/10.1161/CIRCRESAHA.120.317134. Online ahead of print.

7. Ingraham NE, Barakat AG, Reilkoff R, Bezdicek T, Schacker T, Chipman JG, et al. Understanding the Renin-AngiotensinAldosterone-SARS-CoV-Axis: A Comprehensive Review. Eur Respir J [Internet]. 2020:2000912. Disponible en: http://erj.ersjournals.com/lookup/doi/10.1183/13993003.009122020.

8. D’Ardes D, Boccatonda A, Rossi I, Guagnano MT, Santilli F, Cipollone F, et al. COVID-19 and RAS: Unravelling an Unclear Relationship. Int J Mol Sci. 2020;21:3003.

9. Schiffrin EL, Flack J, Ito S, Muntner P, Webb C. Hypertension and COVID-19. Am J Hypertens [Internet]. 2020;33:373-4. Disponible en: https: / /academic.oup.com/ajh/article/33/5/373/5816609.

10. Mancia G, Rea F, Ludergnani M, Apolone G, Corrao G. ReninAngiotensin-Aldosterone System Blockers and the Risk of Covid-19. N Engl J Med [Internet]. 2020:1-10. Disponible en: http://www.ncbi.nlm.nih.gov/pubmed/32356627.

11. Reynolds HR, Adhikari S, Pulgarin C, Troxel AB, Iturrate E, Johnson SB, et al. Renin-Angiotensin-Aldosterone System Inhibitors and Risk of Covid-19. N Engl J Med [Internet]. 2020:1-8. Disponible en: http://www.ncbi.nlm.nih.gov/pubmed/32356628. 